\title{
RECENT TECHNIQUES IN SOLAR OPTICAL ASTRONOMY FROM SPACE
}

\author{
E. M. REEVES \\ (Harvard College Observatory, Cambridge, Mass., U.S.A.)
}

\begin{abstract}
The article summarizes some of the recent advances in the techniques of solar space research, particularly over the last 4 years, in an attempt to review the current state of instrument technology. The present state of development in rocket and satellite vehicles for solar observations, far ultraviolet detectors, optical materials, ultraviolet reflection coatings, filters, and photographic film fogging, are among the topics described.
\end{abstract}

\section{Introduction}

The experimental objectives of solar space research frequently follow the general trend towards ever-increasing spectral resolution and photometric accuracy coupled, whenever possible, with spatial resolution on the disk. The experiment flux is frequently limited by the attempt to achieve these aims simultaneously, rather than limited by instrumental diffraction effects, and then one seeks ways to obtain sufficiently large collecting apertures to achieve the best compromise between spatial and spectral resolution. The advances in technology useful in this process are improvements in pointing controls, optical efficiencies, and detector efficiencies. Only rarely are solar satellite experiments limited by telemetry capabilities since these purely electronic areas are managing to keep up with or ahead of available experiments. This will shortly begin to change, however, and various forms of data compression techniques will be required for solar instrumentation. This problem becomes especially critical with the development of photoelectric image storage devices with storage capabilities for large amounts of data, as is currently the case for the simple photographic plate.

Since the last General Assembly of the IAU in 1964 there have been two launches of satellites for solar optical astronomy. These are the OSO-II and OSO-III Orbiting Solar Observatories, which were launched in February 1965 and March 1967. Figure 1 shows the general arrangement of the OSO-IV, which is a gyroscopically stabilized wheel containing a number of experiments that view the Sun once per revolution of $2 \mathrm{sec}$. Two or more experiments are carried in the sail portion, which contains the solar cells for spacecraft power and orients the instruments to the centre of the solar disk to an accuracy of approximately $20 \mathrm{sec}$ of arc. Data are transmitted to the ground in real time and also recorded on magnetic tape in the satellite and played back to the ground station, on command, once per orbit.

Perek (ed.), Highlights of Astronomy, 75-93. (O I.A.U. 


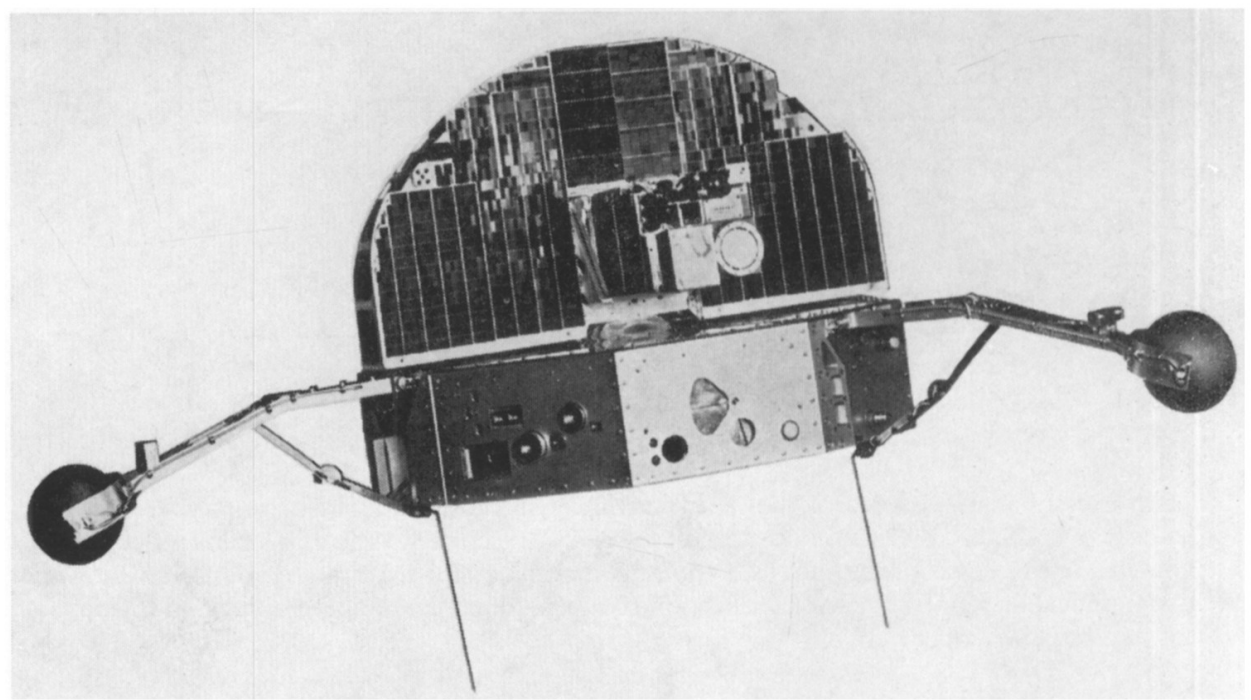

FIG. 1. General arrangement of an Orbiting Solar Observatory spacecraft.

Unlike the first OSO (March 1962), which pointed the experiments at the centre of the Sun, the second OSO satellite provided a raster pattern of about 40 min of arc on the side, as indicated in Figure 2. The scientific instruments in the pointed section had a field of view of about $1 \mathrm{~min}$ of arc, and the raster pattern of the spacecraft was used to build up spectroheliograms with this resolution every $5 \mathrm{~min}$. One of the next OSO's, namely OSO-G, will be provided with an additional small raster pattern of 7 arc minutes square which will provide $30 \mathrm{sec}$ of arc spatial resolution images in $30 \mathrm{sec}$, and will also provide the first opportunity for 'offset pointing' to any location on the solar disk to permit wavelength scans from selected solar formations. These and other improvements in the capabilities of solar satellites are the cornerstones for the design of scientific instruments and provide the bases for a wider range in the type of solar optical observations which can be made.

\section{Pointing Controls For Rockets}

For quite a number of years, sounding rocket experimentation for the solar ultraviolet has been well served with the biaxial pointing control, which is unstabilized in roll angle about the solar vector but provides quite reasonable pointing accuracies to the Sun's centre in the range of 1-2 min of arc.

Just a few weeks before the last IAU Assembly in Hamburg, a group of British experimenters under the direction of R. Wilson successfully flew an Elliott Bros. 3-axis stabilized rocket in which the roll angle was controlled by magnetometer error signals. The system achieved better than $10 \mathrm{~min}$ of arc of pointing in pitch and yaw, 


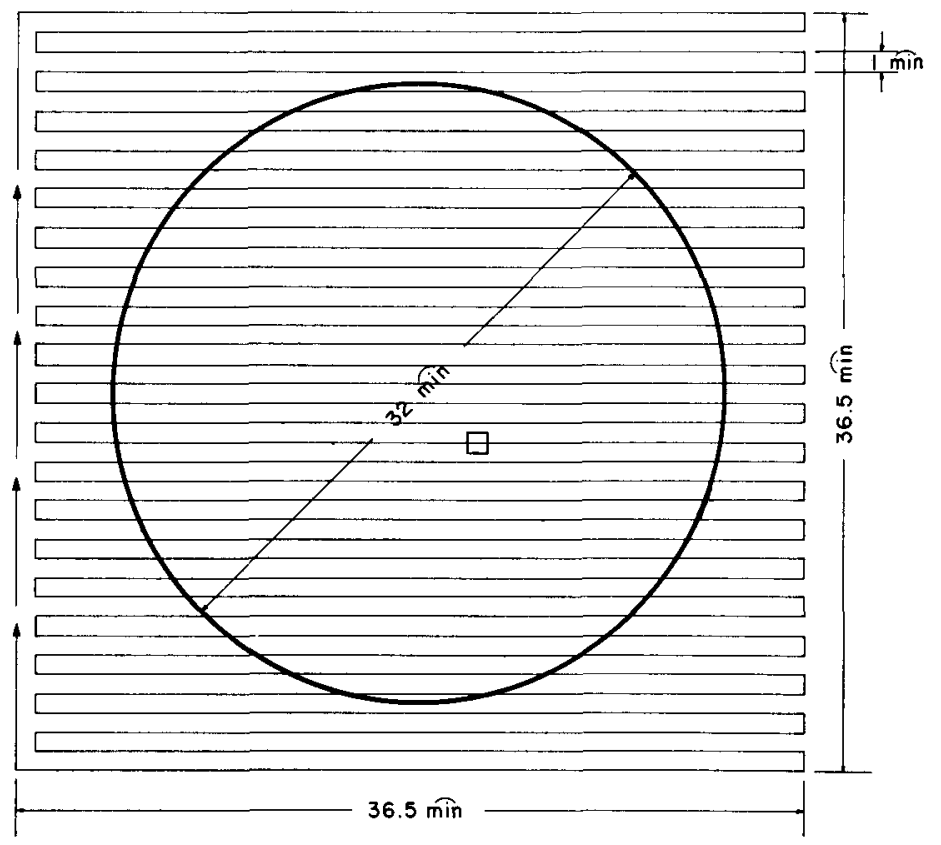

FIG. 2. The raster pattern of OSO spacecraft for generating spectroheliograms.

and a roll stability of approximately $10^{\circ}$ peak to peak. To provide greater accuracy in pitch and yaw, the experiment telescope mirror was servo-controlled with a secondary mirror and split field solar sensor, stabilizing the solar image to several seconds of arc. The optical arrangement is shown in Figure 3. A significant achievement resulted from the development of this system when Dr. Wilson's group obtained the chromospheric spectrum in April 1965 by setting the spectrograph slit about $10 \mathrm{sec}$ of arc outside the visible limb with a slit width of $4 \mathrm{sec}$ of arc. The experiment used Kodak Pathé SC-5 and SC-7 Schumann film and recovery techniques (Burton and Wilson, 1965; Burton et al., 1967).

An interesting achievement in pointing control systems has been used by J.-E. Blamont to obtain the time variation in a number of broad bands in the ultraviolet between $1200 \AA$ and $2400 \AA$ during the progress of the November 12, 1966, eclipse in Argentina. The French system was launched in a Titus rocket from a portable range established for the purpose in Argentina with primary data by telemetry, although provision was made for photographic recovery for other experiments. The experiments were aligned along the axis of the rocket and a system of nitrogen gas jets and pre-set gyroscopes was used to align the instruments with the Sun. Prior to totality a solar sensor provided information to correct the gyroscopes, which then kept the rocket aligned as the Moon provided the complete eclipse. The system was capable of achieving pitch and yaw accuracies of approximately $15 \mathrm{sec}$ of arc and a roll rate of 


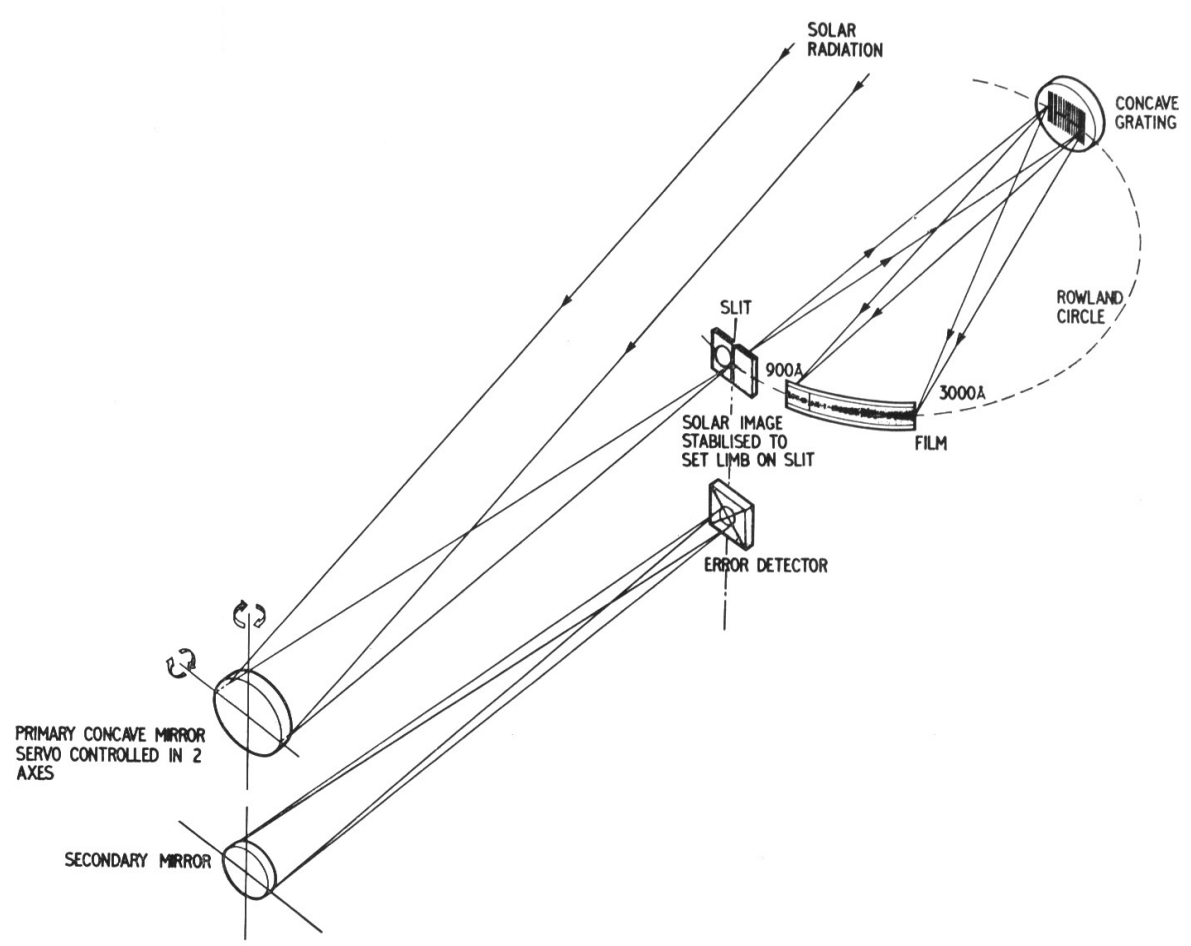

FIG. 3. Diagram of the optical system for the Culham-rocket experiment.

$15 \mathrm{sec}$ of arc/sec. During a total eclipse of this type it is possible to obtain spatial resolutions of $1 \mathrm{sec}$ of arc or better, limited by the Moon's irregularities. With control of the precise roll angle and selection of a smooth lunar area it would be possible to achieve spatial resolution in the order of $0.1 \mathrm{sec}$ of arc.

Several 3-axis pointing control systems are nearing completion in the U.S.A.; they will provide pitch and yaw accuracies of better than $15 \mathrm{sec}$ of arc and absolute roll position to several degrees for both solar and stellar observations. Three different systems with somewhat different characteristics have been assembled and tested and are scheduled for test launch in the early autumn. Two of the systems, SPARCS and STRAP, are produced by NASA and the SPCS system is available from Ball Bros. Research Corporation. A summary of the pointing control characteristics is contained in the next review paper (Wilson, 1968).

\section{Detectors}

The activity in the field of space research has continued to demand the development of improved photomultiplier tubes for the ultraviolet and vacuum ultraviolet regions which are capable of withstanding the launch environments of a variety of rockets. 
Photomultipliers for the solar ultraviolet fall into two general classes, the very shortwavelength, windowless devices, and the more conventional discrete stage electronmultiplier tubes with a variety of window and photocathode materials. These latter photomultipliers are now available with a wide range of photocathodes such as $\mathrm{KBr}$, $\mathrm{CsBr}, \mathrm{RbI}$ and CsI, CsTe, etc., with ultraviolet window materials such as glass, quartz, sapphire, lithium fluoride, and most recently magnesium fluoride. $\mathrm{MgF}_{2}$ windows represent a significant improvement over LiF in that they can accept a better optical polish, are not susceptible to humidity, are harder, and exhibit a greatly reduced sensitivity to both ultraviolet and energetic particle-radiation damage, while allowing comparable efficiencies above $\lambda 1150 \AA$.

The radiation damage indicated in Figure 4 for $\mathrm{MgF}_{2}$ and $\mathrm{LiF}$ corresponds to a predicted maximum flux for January 1966 in a circular polar orbit at $1400 \mathrm{~km}$. The transmissions were measured within hours of radiation and all radiated crystals showed fluorescence which was not detected visually. Of the materials tested, synthetic
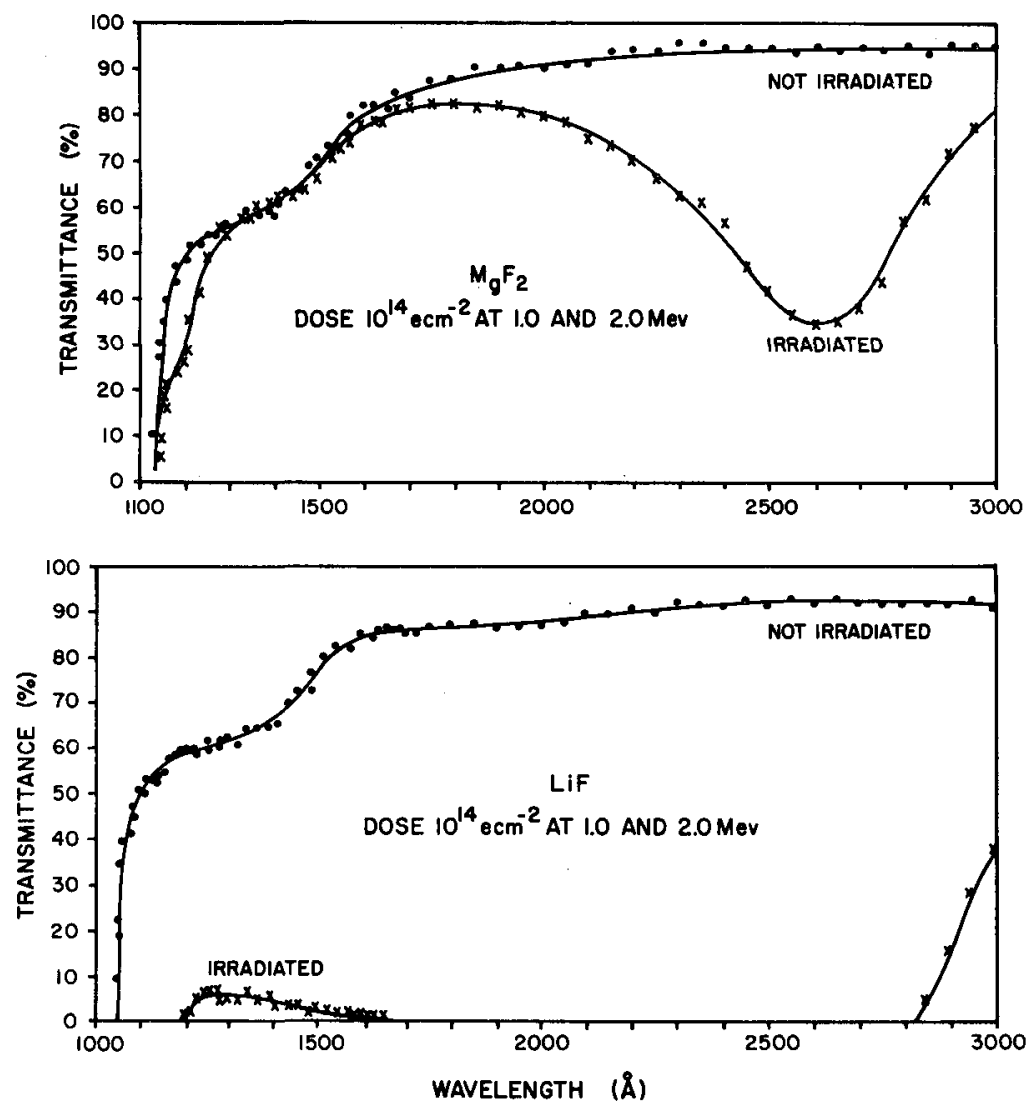

FIG. 4. Effect of high-energy electron bombardment on the transmittance of $\mathrm{MgF}_{2}$ and LiF. 
sapphire and quartz of highest purity showed the least degradation of transmittance with irradiation (Heath and Sacher, 1966). I will not refer in greater detail to the large amount of work which has been done on the radiation effects on glasses for lenses for space applications, since a number of review articles are available (Monk, 1952; Kreidl and Hensler, 1957; Stroud, 1962; Becker, 1967). A wide selection of optical materials is now available in the visible region of the spectrum which do not show appreciable effects from hard radiation in space. A number of optical systems, particularly in the Moon and planetary satellites, have survived long periods in space without appreciable degradation, provided that due care has been exercised in the choice of materials.

One significant improvement in these more conventional photomultipliers for the ultraviolet has been made by Electro-Mechanical Research, Inc. (Figure 5), in which

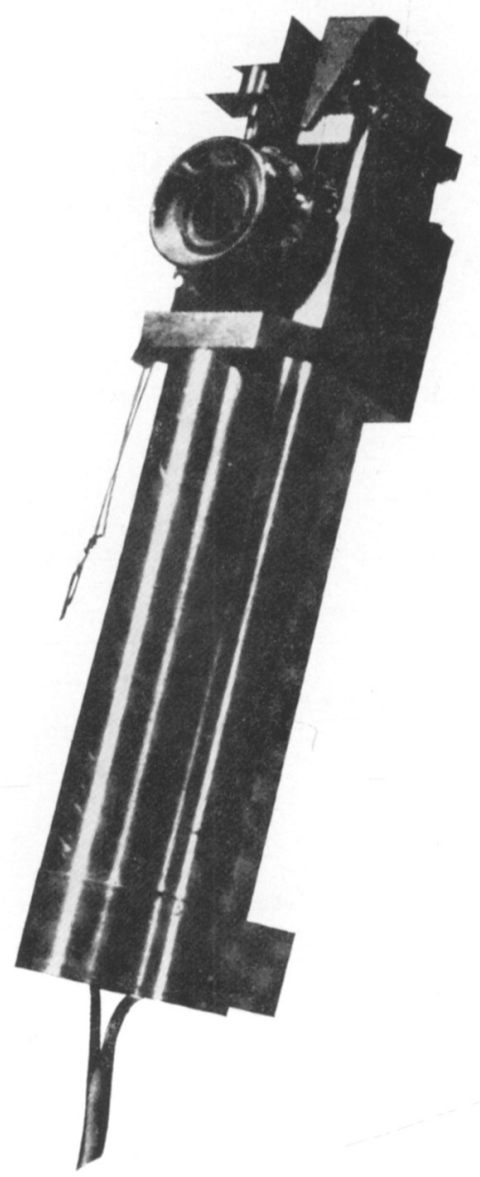

FIG. 5. A photomultiplier with removable side window. 
the window and its housing can be removed in flight in $50 \mathrm{~ms}$ by the action of an explosive device, as a once only and irreversible process (Rome, 1964). The conversion of the multiplier from a windowed to a windowless device, once a clean high vacuum has been achieved, has some useful advantages. The inability to remove and replace the window during photometric calibration is a limitation insofar as the detector efficiency cannot be verified during instrument testing. There is no reason why this type of electron multiplier could not be fitted with a high-work function metal cathode, blind above $1300 \AA$, and still have the very large dynamic range of several megahertz, characteristic of the discrete dynode multiplier.

A photon scintillating device has been developed by the same company and by other laboratories as well, and is illustrated in Figure 6 (Rome, 1964). The incident
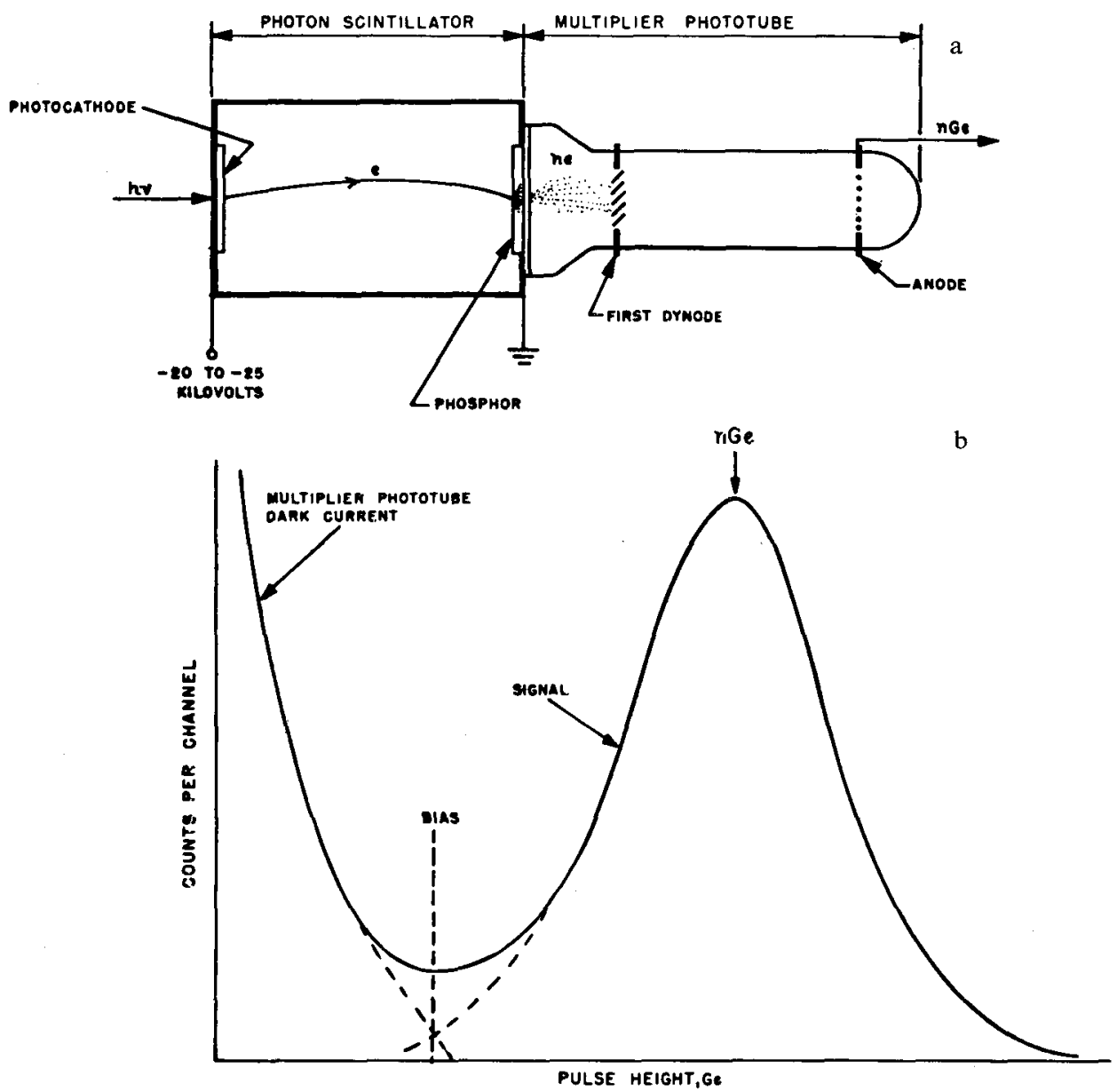

Fig. 6. (a) Photon scintillator detector assembly. (b) Pulse-height distribution. 
photon releases electrons from a semi-transparent photocathode, which are then accelerated by approximately $20 \mathrm{KV}$ and strike the phosphor with sufficient energy to release a large number of photons within the fluorescence time of the phosphor. The converted photons are then viewed with a conventional photomultiplier. The advantage in this device lies in the output pulse-height distribution, in which the signal exhibits a well-defined peak which permits discrimination against noise pulses in the photomultiplier. The efficiency of detection is increased over that of a single ultraviolet multiplier where the pulse-height distribution requires that some counts be biased off. The device could also be used with a side window or as a windowless device with a metal photocathode, although these latter would require some special development.

The common EUV photomultiplier for space research is the crossed electric and magnetic-field multiplier with continuous dynode strips (Timothy et al., 1967; Hinteregger, 1961). These devices are produced by the Bendix Company and are illustrated in Figure 7. They are generally referred to as Magnetic Electron Multipliers or MEM's. The tubes are usually provided with a metal photocathode such as tungsten or other inert metal, all of which are blind above $1300 \AA$ (Cairns and Samson, 1966) and sensitive down to the $\mathrm{X}$-ray region. The devices consume little power, and are capable of providing a linear dynamic range up to about $200 \mathrm{KHz}$. The continuous resistance dynode and field strips are quite sensitive to temperature which produces changes in the gain of the multiplier. Unless the detection system as a whole has been designed to compensate for the effect, which can be accomplished in one of several ways, the detector efficiency will be temperature-dependent. The devices are functionally quite good, although a considerable amount of work is still required to fully understand their properties and to properly qualify them for extended satellite use with constant efficiency.

The Channel Electron Multiplier (CEM) has received much attention and development for rocket and satellite use (Adams and Manley, 1965; Goodrich and Wiley, 1962; O'Brien et al., 1967). A variety of different designs with and without shaped cathode ends as produced by Bendix are shown in Figure 8. Similar devices are now available from Electro-Mechanical Research, Inc. in the U.S.A. and from Mullard in England. The devices are a hollow tube coated with a resistive material which serves as the dynode and also as the cathode material. The photometric response of the channel multipliers is quite similar to that of the metal photocathodes for the region above $500 \AA$ (Johnson, 1966). Primary photoelectrons are accelerated along the tube by the $3-K V$ potential, and on striking the wall they release additional secondary electrons, which are in turn accelerated to give a high-gain output pulse. The channeltrons are basically very simple and reliable, although guaranteed linear dynamic range is limited to about $20-60 \mathrm{KHz}$. The tubes are frequently operated at highest gain so that the output pulses 'saturate' the multiplier, which provides a natural shaping of the anode pulses. However, for the higher intensities this mode leads to the problem 


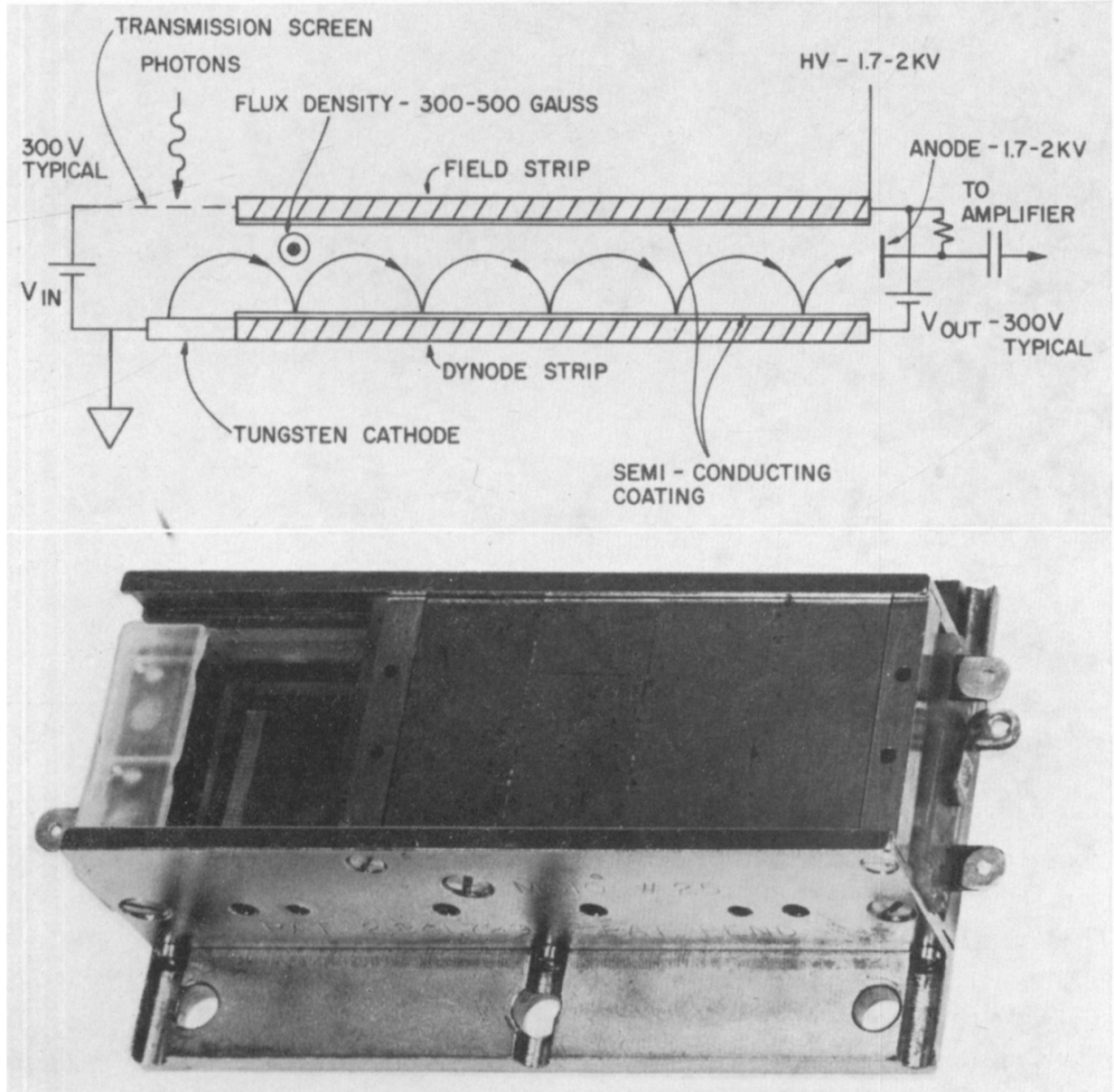

FIG. 7. Bendix crossed electric and magnetic-field photomultiplier (MEM).

of 'fatigue', which shows up as a deterioration in the pulse height distribution which becomes more critical as the intensity $\times$ time product increases. This can be overcome to some extent by using the multiplier at lower gains.

A further development in the simple channel multiplier has been the serial use of two channels of different impedances. The lower-impedance second stage allows more power to be delivered, and the linear dynamic range can be increased to about 1-2 $\mathrm{MHz}$ for random pulses. These devices are in the developmental stage at the present time.

One of the most interesting advancements in the field of EUV detectors has been the miniaturization and close packing of arrays of channel multipliers. Bundles of multipliers well in excess of 15000 have been produced with capillary size of $250 \mu \mathrm{m}$ and 


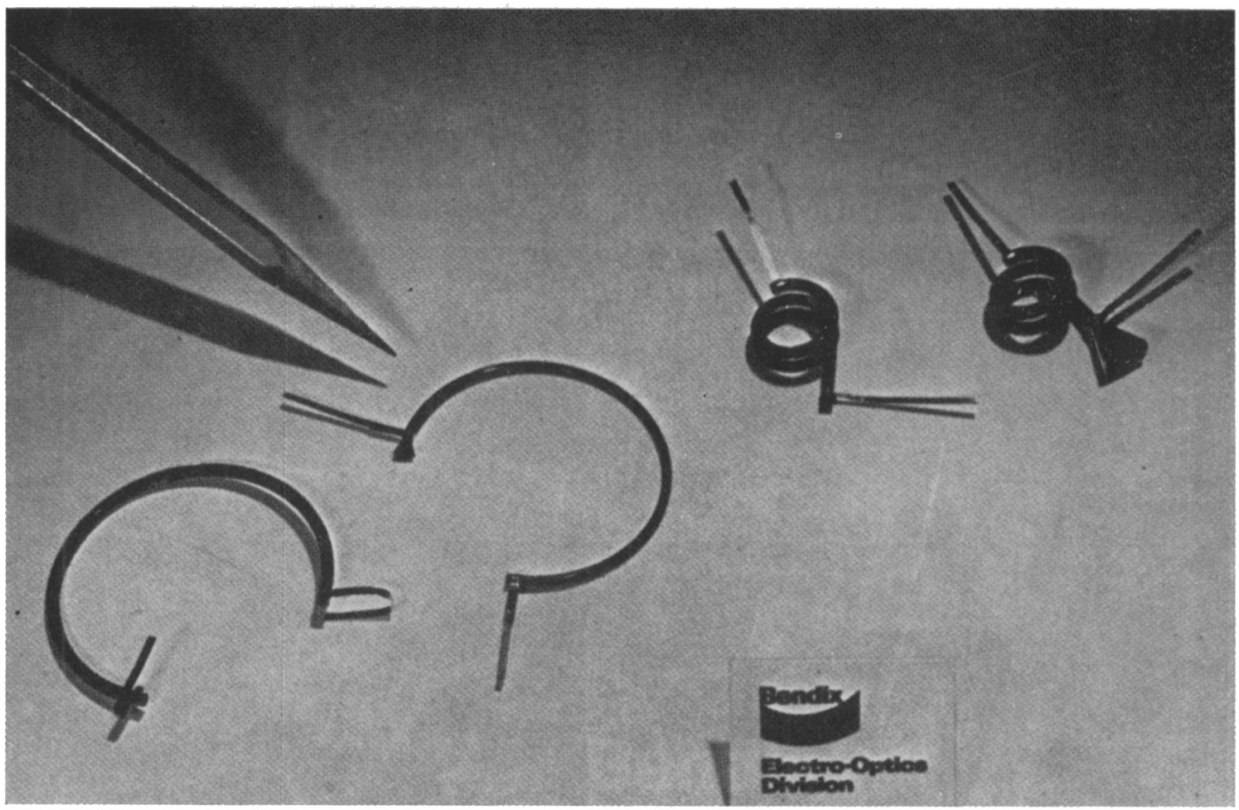

FIG. 8. Several varieties of Bendix Channeltron electron multipliers.

even with multiplier tubes as small as $12 \mu \mathrm{m}$ or less. For each photoelectron resulting from a photon incident on the face of the array, an amplified electron pulse emerges from the anode end with the spatial intensity distribution on the front end preserved. These electron clouds may then be accelerated with a potential of 8-10 KV to strike an aluminized phosphor and film device which will produce a photographic image spot for a single initial photoelectron, or the array can be used with some comparable electronic imaging system. At the present time these arrays are commercially available as a special order.

\section{Optical}

Above $1200 \AA$ optical efficiencies can be obtained which are quite comparable to visible region reflectivities, and folded optical systems can be used to reduce aberrations and decrease the instrument envelope. The most widely used coating is still aluminum with a thin layer (200-400 $\AA$ ) of magnesium fluoride deposited in high vacuum immediately following the deposition of aluminum. This prevents the formation of an aluminum oxide layer. Efficiencies between 80 and $90 \%$ can be obtained over broad wavelength ranges by varying the thickness of $\mathrm{MgF}_{2}$ overcoating (Berning et al., 1960; Bates and Bradley, 1967; Hass and Ritter, 1967).

Multilayer coatings for the visible region have certainly progressed in the last few years, particularly for narrow-band transmission filters and non-reflective coatings. At Harvard College Observatory we are currently evaluating $\mathrm{H} \alpha$ interference filters for 


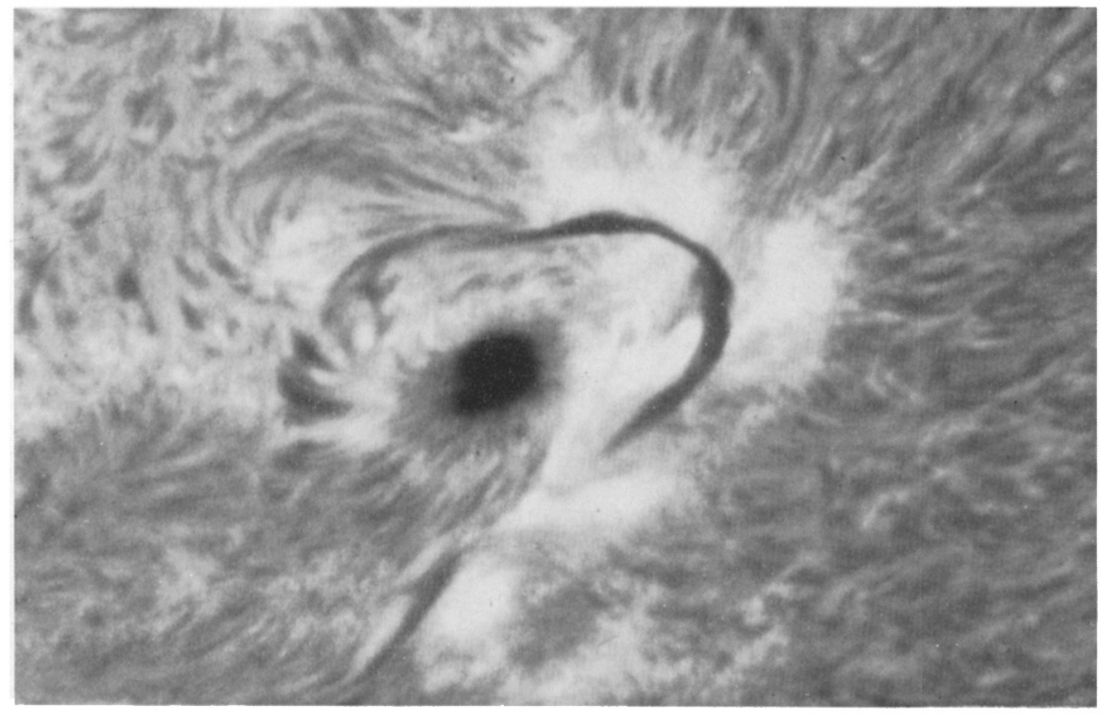

\section{$\Delta \lambda=0.5 \AA$ HALLE FILTER}

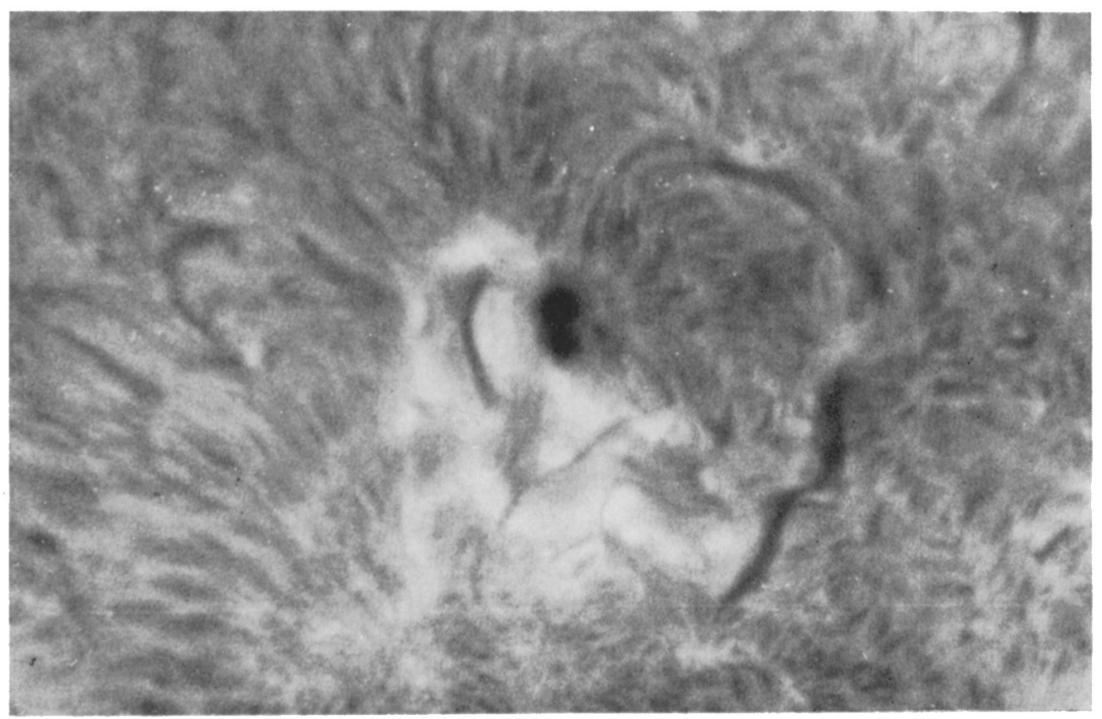

\section{$\Delta \lambda=0.6 \AA \quad$ TYPE "U" FILTER}

FIG. 9. Photographs of similar solar active regions using a Halle birefringent and a type ' $U$ ' interference filter. (Photographs courtesy of Lockheed Solar Observatory.) 
satellite applications. Spectrolab in California are now able to produce narrow-band filters with a halfwidth of $0.5 \AA$, a uniform field, and transmissions of $20-25 \%$. The filters have been produced down to $0 \cdot 3 \AA$ halfwidth with a transmission of more than $10 \%$. Several other companies are also engaged in developing similar filters and within the next few months should have been successful. The Spectrolab type ' $U$ ' filters are a solid Fabry-Pérot device with interference coatings on each side of a thin substrate. As a result the devices are only a few millimeters thick and are structurally rugged. They are thus much more compact than the birefringent filters, very insensitive to shock and vibration, and have a lower requirement on temperature stabilization.

Figure 9 shows two pictures taken by the Lockheed Solar Observatory of two solar regions, one with an $\mathrm{H} \alpha$ Halle filter and the other with a similar bandpass interference filter. The spatial resolutions are quite comparable and are about $1 \mathrm{sec}$ of arc or a little better. The interference filters have significantly greater transmission than the birefringent filters, although at the present time they are somewhat more limited in the field of view. A report on the progress in this filter evaluation program should be available in a few months. Although no difficulty would be expected, interference filters have not been qualified for extended exposure to high vacuum, but there would be no problem in flying the filters in a sealed, thermostatically controlled oven. Our group at Harvard plans to do this on one of the early manned solar satellites to provide the astronaut with a solar pointing capability using $H \alpha$. Radiation damage in the filters does not appear to be a problem.

There have been some advances in making interference filters for the region below 23000. Bates and Bradley (1966) have made filters with $300 \AA$ passbands or less and a transmission of $25 \%$ for the region $1800-2400 \AA$. They are using the filters in highresolution ultraviolet rocket and satellite interferometers for the Sun.

Miss Fredga (1966) has recently obtained photographs of the Sun in Mg II at 2802.7 $\AA$ from a rocket in which a specially developed Solc-type birefringent filter was combined with a telescope and 35-mm camera. The filter had a passband of $4 \AA$ and transmission of $1 \%$ or less. The filter also required the development of a servo-controlled temperature system to keep the filter on line. A schematic diagram of the equipment is shown in Figure 10. This does represent the first time that a birefringent filter has been flown*in a rocket to obtain pictures in the ultraviolet. Figure 11 shows a reproduction of the results of the flight. The image resolution is approximately $1 \mathrm{~min}$ of arc and was limited by jitter on the rocket solar-pointing control system. The development and qualification of these narrowband birefringent filters for the ultraviolet is a significant achievement, and pictures at higher spatial resolution will be possible now that improvements in solar-pointing controls are available.

In the visible region of the spectrum the adoption of the multiple occulting disks in white-light coronagraphs, as suggested by Newkirk, has significantly reduced the problem of diffraction-scattered light in balloon- and rocket-borne coronagraphs (Newkirk and Bohlin, 1964). 


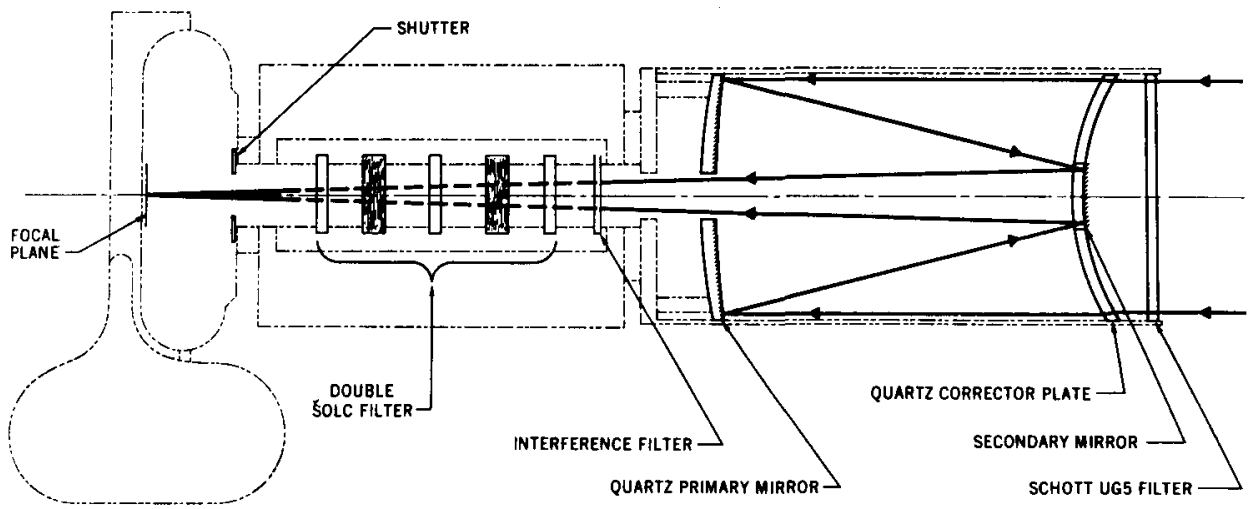

FIG. 10. Optical system for a MgII rocket spectroheliograph.

12 APRIL 1965

N

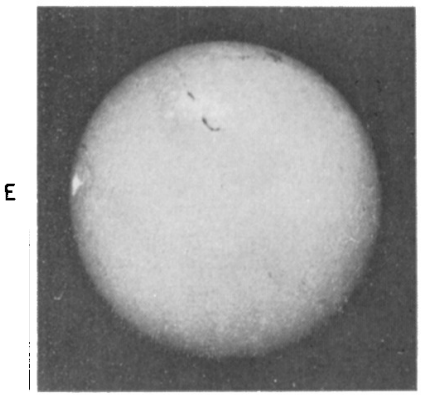

Ha $14: 49$ UT
N

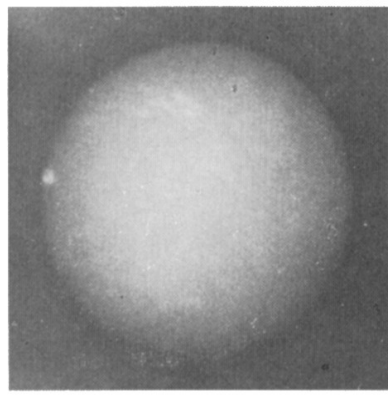

$\mathrm{Mg}$ 14:54 UT
$\mathrm{N}$

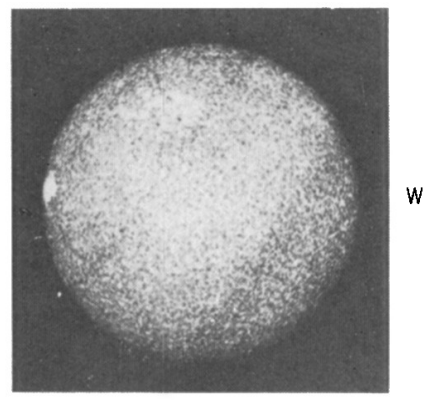

Cak 14:41 UT

FIG. 11. Comparison of the MgII with hydrogen and calcium spectroheliograms.

For the wavelength region below $1200 \AA$ at near normal incidence, reflectivities are substantially lower than can be obtained above $\lambda 1200$ with $\mathrm{Al}$ and $\mathrm{MgF}_{2}$. The best available coatings are still Au (Canfield et al., 1964), Pt (Reeves, unpublished), and more recently, Ir (Hass et al., 1967).

Figure 12 shows the reflectivities for $\mathrm{Au}, \mathrm{Pt}$ and $\mathrm{Ir}$ from $300 \AA$ to $2200 \AA$. An additional edge which had been noted in $\mathrm{Au}$ has also been found very recently near $400 \AA$ in both Pt and Ir (Hunter, private communication). Hunter's most recent paper on Ir contains data only down to $\lambda 500$, although I have indicated by a dotted line the approximate shape of the curve to $300 \AA$. The $400 \AA$ edge in Pt does not seem to be as pronounced as it is in Au.

The reflecting films which give the maximum reflectance are partially transparent rather than opaque and are quite sensitive to small amounts of contamination, which greatly reduces the reflectance; however, cleaning will usually restore the initial reflec- 


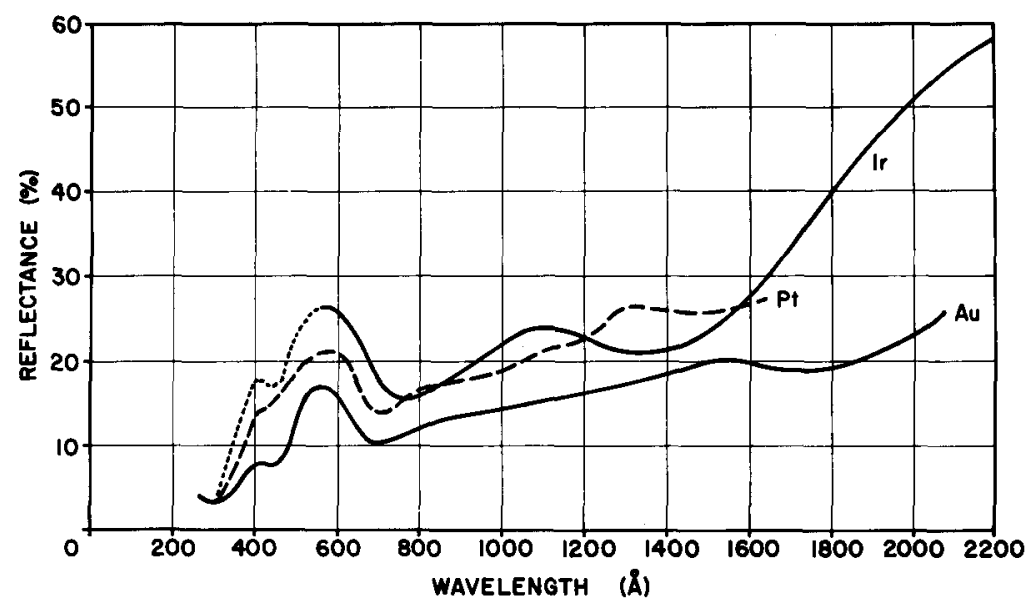

FIG. 12. Reflectances of $A u, P t$, and Ir in the EUV.

tance, if ultraviolet radiation has not been too intense. The techniques of preparation are critical, and there is some difficulty in reliably obtaining mirrors with these reported reflectances.

There has been considerable concern during the last 5 years or so over the use of replica gratings for extended-lifetime satellites. The use of replica gratings for space instruments stems not only from the expense and scarcity of original gratings, but also from the fact that, in some cases, the performance of replica gratings exceeds that of the ruled original, both in increased signal and decreased scattered light. Some initial experiments performed a few years ago indicated that failure of the replicating plastic layer resulted from exposure to a simulated space environment. Some six groups in the U.S.A. have been carrying out research programs to obtain better information on the effects of thermal cycling from $-20^{\circ} \mathrm{C}$ to $+50^{\circ} \mathrm{C}$ at pressures of less than $10^{-8}$ Torr, irradiation with $1.5 \mathrm{MeV}$ electrons at dose rates up to $10^{15} \mathrm{~cm}^{-2}$, and limited use in a 600 nautical mile polar-orbit satellite. All of the present data, although they are by no means complete, indicate that replica gratings can be qualified for use in satellite instruments (Paul et al., 1967).

The problems associated with optical systems in space are generally quite well known. A recent paper by Becker (1967) reviews some of the general problem areas: outgassing contaminations, meteroid damage, radiation, and temperature effects. The thermal problem is one which is of special importance for solar satellites, particularly in near-equatorial orbits where the Sun's radiation is occulted every revolution, and where the time-variant Earth albedo can cause a transverse bending of the optical system. These problems become particularly important when high spatial and spectral resolutions are required. Up to the present time, however, solar satellite EUV observations have been limited to about $1 \mathrm{~min}$ of arc spatial resolution in telescope systems; 
here the problems are less severe, and normal optical materials can be used with passive thermal control. In rocket flights, significantly higher spatial and spectral resolutions have been achieved, since thermal distortions can generally be overcome during the short period of a sounding rocket flight by a certain amount of careful design and selection of compensating materials for construction.

For the larger solar satellites now being designed and built, thermal considerations play one of the most important roles of all in instrument design. The heating of the front surface of a mirror at orbital sunrise sets up a time-varying thermal gradient through the mirror blank which causes a change in curvature. Even in the steady state, the change in curvature $(\gamma)$ is $\gamma=q(\alpha / k)$, where $\gamma$ is the change in curvature, $q$ is the heat input per unit area, $\alpha$ is the thermal expansion coefficient, and $k$ is the thermal conductivity; the resulting change in focal length $(\Delta f)$ is given approximately by $\Delta f \approx 2 \gamma f^{2}$, where $f$ is the focal length.

In the ultraviolet particularly, the reflective coating must be optimized for that particular region, with little or no control over the visible and infrared absorption coeffcient which determines the heat input. For the $\mathrm{Al}$ and $\mathrm{MgF}_{2}$ films, this effective absorption coefficient is only $\mathbf{7 . 6 \%}$, but for the EUV semi-transparent Pt films it has recently been measured by us to be $0.32 \pm 0.02$ on one particular sample. This absorption coefficient is now being evaluated for various EUV coatings in our own laboratory.

The ratio of the coefficient of thermal expansion $(\alpha)$ to the thermal conductivity $(k)$ determines the susceptibility of the material to distortion effects, and this ratio must be kept small either by having a large thermal conductivity or a small expansion coefficient (see Table 1). For the larger solar telescope mirrors, the use of fused silica or quartz may be completely unsatisfactory, unless somewhat elaborate steps are taken to increase the effective thermal conductivity artificially.

\section{Table 1}

\section{Selected parameters for the selection of materials for telescope mirror substrates}

\begin{tabular}{llcc} 
Material & \multicolumn{1}{c}{$\alpha\left(\times 10^{-7}{ }^{\circ} \mathrm{C}\right)$} & $\mathrm{k}\left(\right.$ Watts $\left.\mathrm{cm}^{-1}{ }^{\circ} \mathrm{C}^{-1}\right)$ & $\alpha / \mathrm{k}\left(\times 10^{-7}\right)$ \\
Quartz & 5.5 & 0.0138 & 400 \\
$\mathrm{Be}$ & 124 & 1.58 & 78.5 \\
$\mathrm{Cu}$ & 166 & 3.92 & $42 \cdot 3$ \\
ULE Silica & $0.2 \pm 0.3\left(5-35^{\circ} \mathrm{C}\right)$ & 0.0138 & 14.5 \\
Cer-Vit & $0 \pm \mathbf{0 . 3}\left(0-50^{\circ} \mathrm{C}\right)$ & 0.0167 & $0\left(30^{\circ} \mathrm{C}\right)$
\end{tabular}

The approach used on the Mariner-IV television system (which, however, did not have the high incident solar heating directly through the optical system) was to match the mirror material and the support structure so that the image stayed within the depth of focus as the thermal environment changed. The method requires material of 
high thermal conductivity in order to reduce temperature gradients. This approach has many advantages; however, the use of metal mirrors such as beryllium requires an overcoating of a hard layer of a material like Kanigen, which is susceptible to 'crazing' or cracking, which gives rise to increased scattered light and distortion of the figure.

Recent experiments by Bennett, Madden, and their co-workers (Bennett, 1963; Bennett and Porteus, 1961 ; Johnston et al., 1967) have measured the effect of surface roughness on scattered light, particularly at the lower wavelengths, at normal incidence. The effect becomes extremely important as the wavelength decreases below $500 \AA$ and may even limit the lower wavelength observed with a mirror. The effect of surface roughness can also be very important in achieving the highest specular reflectance for the EUV region. At the present time, metal mirrors do not appear to be suitable for resolution in the few seconds of arc region in the solar ultraviolet. This does not necessarily hold true for the wavelength region below about $500 \AA$ where one generally uses grazing incidence optical systems with hyperboloids and paraboloids. At grazing incidence, the efficiency of reflection increases markedly, and the tolerances on the figure are reduced. However, the techniques of grazing incidence have specifically been omitted from this review, since they are very similar to those used for soft X-rays and will be treated by other authors during this Joint Discussion.

An alternate approach to the solution of the thermal problem is to make the system insensitive to thermal effects by choosing a material with near zero coefficient of expansion. Three such materials have recently become available; these are: ULE Fused Silica with an expansion coefficient of $0 \cdot 2 \pm 0 \cdot 3 \times 10^{-7}{ }^{\circ} \mathrm{C}^{-1}$, ULE Glass Ceramic with $0 \pm 0 \cdot 1 \times 10^{-7 \circ} \mathrm{C}^{-1}$ made by Corning, and Cer-Vit made by Owens-Illinois. Figure 13 shows a plot of the expansion coefficient vs. temperature for Cer-Vit, which indicates

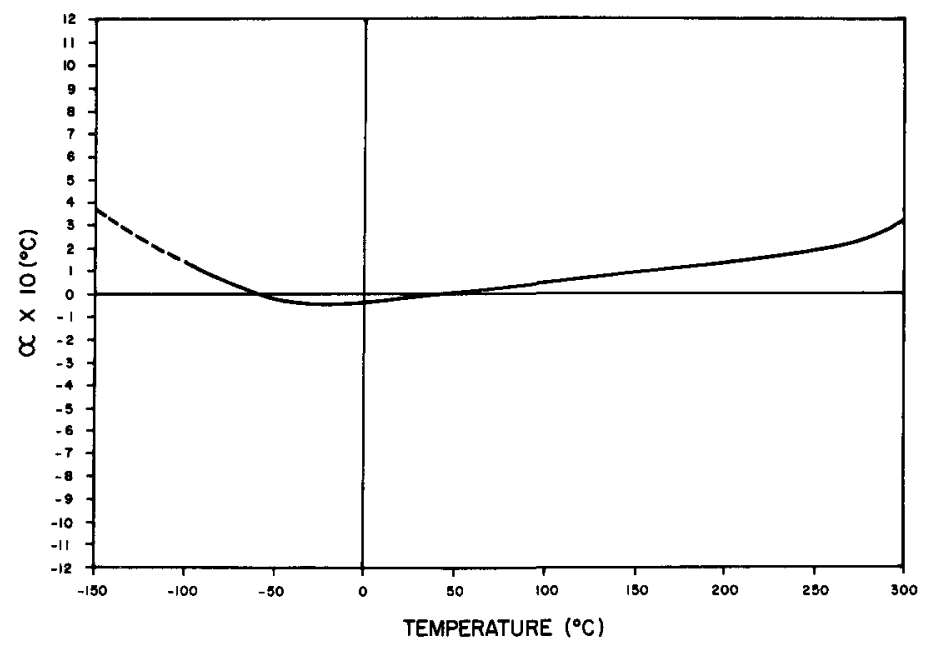

FIG. 13. Coefficient of thermal expansion for Cer-Vit as a function of temperature. 
an expansion coefficient close to zero over a wide temperature range. Recent experiments by Dietz and Bennett (1967) have shown that this new material can be polished as smooth as the best fused silica, namely to an r.m.s. surface roughness of better than $8 \AA$, using a 'Bowl Feed' technique (Dietz and Bennett, 1966). It will also withstand amazing thermal shocks from alternate exposures to ice water and a $350^{\circ} \mathrm{C}$ oven, showing negligible deformation of figure within $\lambda / 10$. These materials will be used for many of the larger solar satellite optics, both for the telescope mirrors and possibly for structural elements for part or all of the instrument assembly.

I have already referred to the complications attendant on the thermal problem at high spatial resolution. An additional problem which currently is limiting many experiments is the use of recoverable photographic film in long-mission satellite experiments. The frequent passage of near equatorial orbit satellites through the lower part of the South-Atlantic Anomaly causes most ultraviolet films, such as the EUV Schumann-type emulsions (SC5, SWR), as well as 103-0, Pan-X, and Plus-X, to be fogged to a density of 0.2 in a matter of a few days. Red-sensitive films such as SO-375 and Starburst films for $\mathrm{H} \propto$ photography are much less prone to radiation fogging. Shielding against the energetic protons responsible does not appear to be possible within reasonable weight constraints. On-board development of the film may have to be used to overcome this problem in long-duration manned missions.

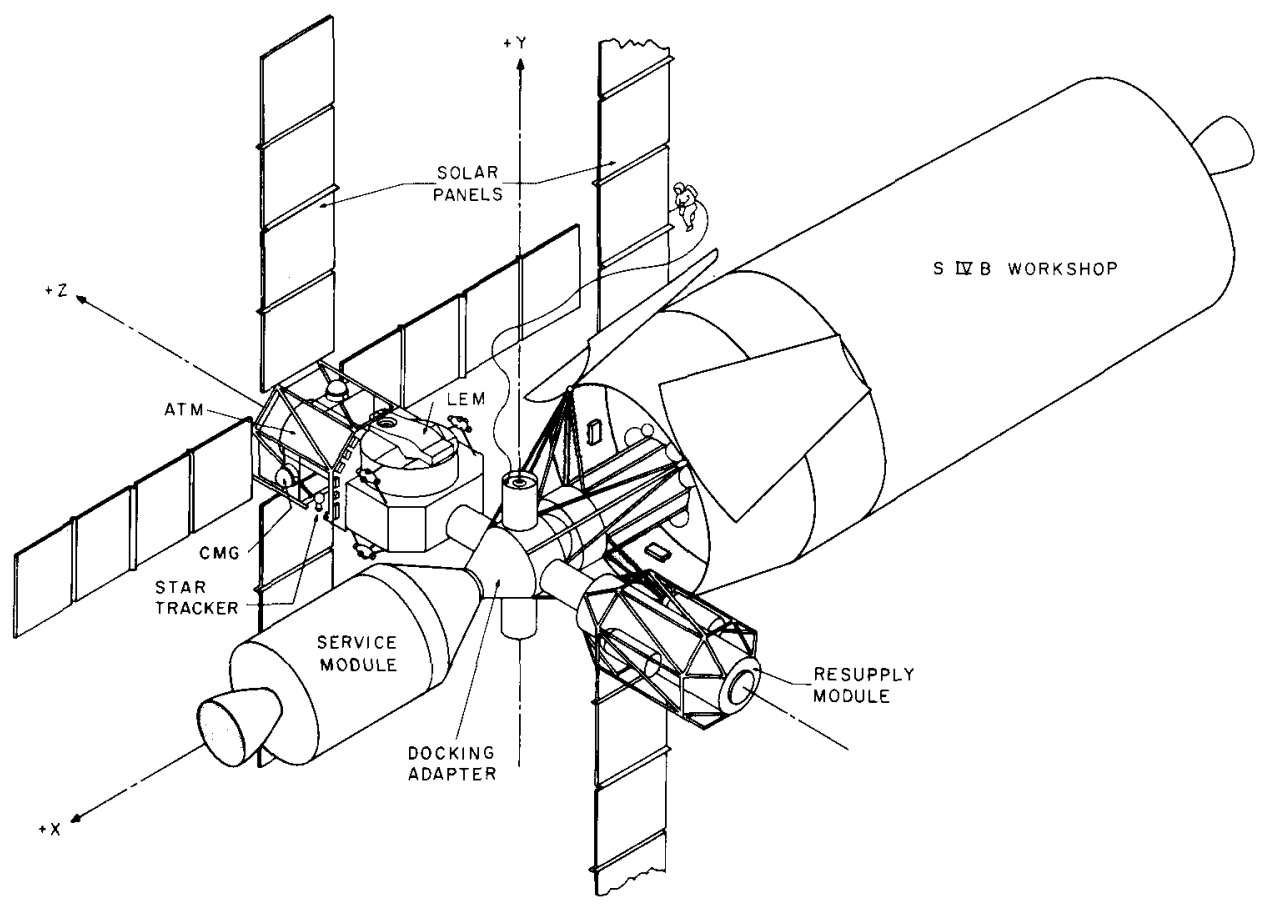

FIG. 14. Illustration of the Apollo Telescope Mount orbited configuration. 
The use of the high data-storage capability of normal photographic emulsions presents many highly desirable advantages; however, their use in satellites with film recovery is presently a large outstanding problem, unless very low or purely equatorial orbits are used, or unless emulsions with lower sensitivity to radiation fogging can be developed. Several research groups in the U.S.A. are investigating the quantitative effects of stimulated anomaly radiation fields on commonly used photographic emulsions (Shelton and deLoach, 1967).

One of the 'techniques', if one can refer to it as such, that will shortly exert a great influence on solar space research is that of manned experiments. Figure 14 shows an illustration of the manned Apollo Telescope Mount (ATM), which is currently being built for launch within the next few years. The ATM contains a group of experiments such as a visible light coronagraph (High Altitude Observatory), X-ray telescopes (American Science \& Engineering and Goddard Space Flight Center), vacuum ultraviolet spectrographs (Naval Research Laboratory), and spectrometers (Harvard College Observatory), EUV photographic (NRL) and photoelectric (HCO) imaging systems, and $\mathrm{H} \alpha$-filter telescopes ( $\mathrm{HCO}$ ). The astronaut will guide the experiments, which will have spatial resolutions of the order of $5 \mathrm{sec}$ of arc, to specific locations on the solar disk to an accuracy of several seconds of arc, using an $\mathrm{H} \alpha$ telescope, and instrument slit-jaw displays with the narrowband interference filter described above. Experiments on the quiet Sun, active regions, and flare activity will then be carried out simultaneously over a wide wavelength range. The advent of the manned solar satellite has introduced a significant new capability into the field of solar space research.

\section{References}

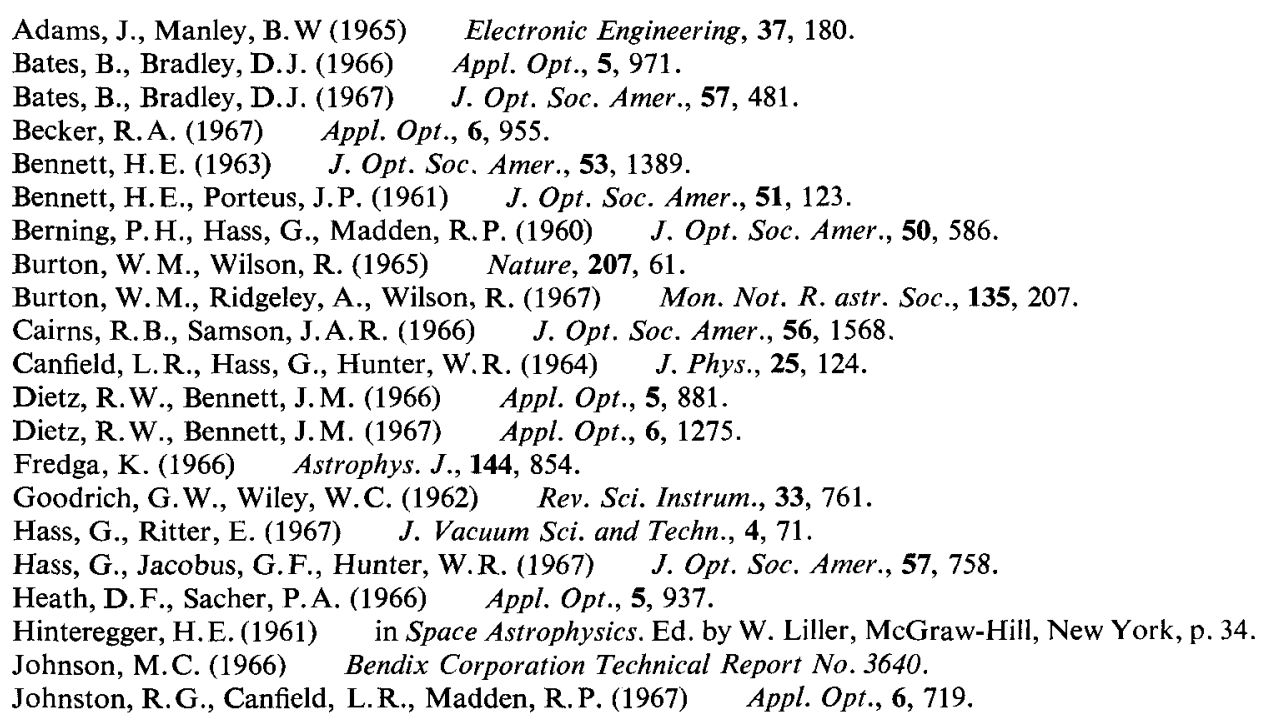


Kreidl, N.J., Hensler, J.R. (1957) J. Opt. Soc. Amer., 47, 73.

Monk, G.S. (1952) Nucleonics, 10, 52.

Newkirk, Jr., G.D., Bohlin, D. (1964) Appl. Opt., 3, 543.

O’Brien, B.J., Abney, F., Burch, J., Harrison, R., LaQuey, L., Wineicki, T. (1967) Rev. Sci. Instrum., 38, 1058.

Paul, F.W., McAndrew, C.E., Diggins, J.C. (1967) J. Opt. Soc. Amer., 57, 582.

Rome, M. (1964a) IEEE Trans. on Nuclear Science, June 1964.

Rome, M. (1964b) Appl. Opt., 5, 855.

Shelton, R.D., deLoach, A.C. (1967) National Aeronautics and Space Administration Report $T M X-53666$. Marshall Space Flight Center.

Stroud, J.S. (1962) J. Chem. Phys., 37, 836.

Timothy, A.F., Timothy, J.G., Willmore, A.P. (1967) Appl. Opt., 6, 1319.

Wilson, R. (1968) in the present volume, p. 94. 